\title{
Assessment of the value of 3D-DSA combined with neurointerventional thrombolysis in the treatment of senile cerebrovascular occlusion
}

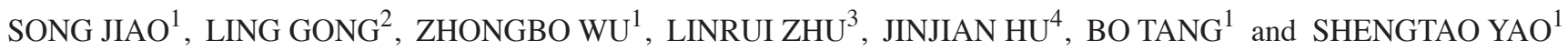 \\ ${ }^{1}$ Department of Cerebrovascular Disease, ${ }^{2}$ Clinical Skill Room and ${ }^{3}$ Tuberculosis Ward, Department of Respiratory Medicine, \\ Affiliated Hospital of Zunyi Medical University, Zunyi, Guizhou 563000; \\ ${ }^{4}$ Department of Neurology, People's Hospital of Xixiu District, Anshun, Anshun 561000, P.R. China
}

Received September 13, 2019; Accepted November 11, 2019

DOI: $10.3892 /$ etm.2019.8274

\begin{abstract}
Assessment of the value of three-dimensional digital subtraction angiography (3D-DSA) combined with neurointerventional thrombolysis in the treatment of senile cerebrovascular occlusion was investigated. A total of 129 patients with senile cerebrovascular occlusion admitted to the Affiliated Hospital of Zunyi Medical University from August 2015 to September 2017 were collected. Among them, 69 patients who underwent neurointerventional catheter thrombolysis under 3D-DSA were included in the study group, and 60 patients treated with neurointerventional thrombolysis were the control group. The levels of inflammatory cytokines IL-6, IL-1 $\beta$ and IL- 8 in the two groups were measured by enzyme linked immunosorbent assay (ELISA) before treatment (T0), 7 days (7d) after treatment (T1) and 14 days (14d) after treatment (T2). The score of the National Institute of Health Stroke Scale and the clinical efficacy of patients in the two groups were compared before and after treatment, and Barthel index (BI) was used for investigation before and after treatment. The recurrence rate of disease in the two groups within 1 year was recorded. At T1, IL-6, IL-1 $\beta$ and IL-8 in the study group were significantly lower than those in the control group $(\mathrm{P}<0.05)$. The NIHSS score in the study group was lower than that in the control group after treatment $(\mathrm{P}<0.05)$. The BI score in the study group was significantly higher than that in the control group after treatment $(\mathrm{P}<0.05)$. After the prognostic follow-up, the disease recurrence rate of the study group was significantly lower than that of the control group $(\mathrm{P}<0.05)$. In conclusion, 3D-DSA combined with
\end{abstract}

Correspondence to: Dr Shengtao Yao, Department of Cerebrovascular Disease, Affiliated Hospital of Zunyi Medical University, 149 Dalian Road, Zunyi, Guizhou 563000, P.R. China E-mail:shg301@163.com

Key words: three-dimensional digital subtraction angiography, neurointerventional thrombolysis, cerebrovascular occlusion, value assessment neurointerventional thrombolysis can significantly reduce the expression of inflammatory cytokines and improve the quality of life in patients with cerebrovascular occlusion, which has a high clinical value.

\section{Introduction}

Cerebrovascular occlusion is a common disease of elderly people, which poses a great threat to human life (1) and has a great impact on patients' quality of life (2). Cerebral infarction is caused by cerebral vascular occlusion, which needs timely opening of the blood vessels to make blood vessel flow normal, thus to achieve the purpose of treatment $(3,4)$. Thrombolysis is a common treatment method for cerebrovascular occlusion in clinical practice and an important way of opening occlusive cerebral vessels (5). With the progress of clinical medicine in recent years, interventional therapy has also been widely used in clinical practice, providing a new method for cerebral vascular occlusion (6). This was confirmed in the studies of Tian et al (7) and Lei et al (8). However, Rao et al (9) achieved a better effect than conventional treatment when they applied interventional therapy to the treatment of aneurysms. Therefore, interventional therapy has gradually become the first choice for clinical treatment of cerebrovascular diseases (10). With the wide application of interventional therapy, however, its disadvantages are gradually exposed. For example, improper selection of puncture site may not only result in ineffective treatment, but also cause aggravation of the disease (11), and it is particularly important for the more complex structure of the brain vascularity when selecting puncture site. Therefore, in order to improve the efficacy of interventional therapy, the relevant auxiliary examinations are the key to treatment.

Three-dimensional digital subtraction angiography (3D-DAS) is an extremely effective detection method (12), which digitizes X-ray images taken before and after the contrast agent to obtain a clear vascular image (13). Compared with traditional X-ray examination, 3D-DAS has the characteristics of high resolution, short examination time and less use of contrast agent, which is particularly suitable for the organization of vascular tissues (14). At present, there are few studies on the application of 3D-DAS combined with 
interventional therapy in the treatment of cerebral vascular occlusion, and its clinical application prospect cannot be determined. Therefore, this investigation provides a reliable reference for the future clinical treatment of such diseases by comparing the use of 3D-DSA combined with interventional therapy and simple interventional therapy for patients with cerebrovascular occlusion.

\section{Patients and methods}

General data. A total of 129 patients with senile cerebrovascular occlusion admitted to the Affiliated Hospital of Zunyi Medical University (Zunyi, China) from August 2015 to September 2017 were collected as the study subjects. Among them, 69 patients who underwent neurointerventional catheter thrombolysis under 3D-DSA were included in the study group, with an average age of $61.6 \pm 4.5$ years. The 60 patients treated with neurointerventional thrombolysis were included in the control group, with an average age of $62.2 \pm 4.8$ years.

The study was approved by the Ethics Committee of Affiliated Hospital of Zunyi Medical University. Patients who participated in this research had complete clinical data. Signed informed consents were obtained from the patients or the guardians.

Inclusion and exclusion criteria. Inclusion criteria: patients met the diagnostic criteria for cerebral vascular embolization, patients diagnosed with cerebral vascular embolization after a series of examinations in the hospital, patients treated in the hospital after diagnosis and patients with complete data and who agreed to cooperate with the investigation of medical staff.

Exclusion criteria: Patients complicated with multiple tumors, patients complicated with other cardiovascular and cerebrovascular diseases, patients complicated with autoimmune diseases, patients suffering from infectious diseases, mental disorders, other organ dysfunction, and physically disabled patients who were unable to take care of themselves.

Therapies. Patients in the control group were punctured in the manner of Seldinger and then placed a guide catheter close to the diseased vessel. The patients were injected with urokinase (Guangdong Techpool Biochemical Medicine Co., Ltd.; H44024032, 10,000 units) 200,000 units + 20 ml normal saline mixture. Fifty milliliters normal saline + 200-500,000 units urokinase was pumped into the patients with a micro-pump catheter at a rate of $1 \mathrm{ml} / \mathrm{min}$. Both groups were re-examined with skull CT $24 \mathrm{~h}$ after treatment. If no bleeding changes were found, patients were given aspirin enteric-coated tablets (Shenyang Original Pharmacolabo Co., Ltd.; SFDA approval no. H20065051, 50 mg/tablet) at a dose of $100 \mathrm{mg}$. Patients in the study group were treated with neurointerventional thrombolysis under 3D-DSA: Local anesthesia was used with the assistance of 3D-DSA (Siemens) to puncture and intubate the femoral artery on the right side of patients, and the artery sheath was inserted. The whole cerebral vascular angiography was performed with a digital subtraction angiography to determine the specific location of the occluded blood vessel, and the subsequent procedure was consistent with the control group.
Detection methods. Enzyme linked immunosorbent assay (ELISA) was used to detect the levels of inflammatory cytokines IL-6, IL-1 $\beta$ and IL-8 in the two groups before treatment (T0), 7 days (7d) after treatment (T1) and 14 days (14d) after treatment (T2). The operation process was strictly in accordance with the kit instructions.

Observational indexes. Main indicators: ELISA was used to detect the levels of inflammatory cytokines IL-6, IL-1 $\beta$ and IL-8 in the two groups before treatment (T0), 7d after treatment (T1) and 14d after treatment (T2). The NIHSS score was used to score the neurological deficits and compare the clinical efficacy of the two groups before and after surgery. The National Institutes of Health Stroke Scale was used for NIHSS score (15). At 14d after treatment, the efficacy assessment standard for stroke was formulated by referring to the " 4 th national academic conference on cerebrovascular diseases' (16). The incidence of adverse reactions after treatment was compared between the two groups.

Secondary observational indexes: Barthel index (BI) was used for investigation before and after treatment (17). Patients in both groups were followed up for 1 year for prognosis, and the recurrence rate of disease in both groups was recorded within 1 year.

Statistical methods. In this study, SPSS 20.0 (IBM Corp.) medical statistical analyzer was used for statistical analysis of collected data. GraphPad Prism 7 (GraphPad Software Co.,Ltd.) was used to image rendering of the collected data. Utilization of enumeration data $(\%)$ was qualified by the Chi-square test and represented by $\chi^{2}$. The measurement data were expressed by mean \pm standard deviation (mean $\pm \mathrm{SD}$ ). All the measurement data were in normal distribution. The independent sample t-test was used for comparison between the two groups, and comparison in group was qualified by paired t-test and expressed by $t$. $\mathrm{P}<0.05$ was considered statistically significant.

\section{Results}

Comparison of general data. There were no differences in sex, age,BMI, nitric oxide sythase (NOS), endothelin-1 (ET-1) pg/ml, vascular endothelial growth factor (VEGF) pg/ml, $50 \mathrm{sec}$ hemodynamics (SRV) $\mathrm{mPa} / \mathrm{sec}$, living environment, smoking history, drinking history, family medical history or ethnicity $(\mathrm{P}>0.05)$ (Table I).

Levels of inflammatory cytokines IL-6, IL-1 $\beta$ and IL-8 of patients in the two groups before treatment (TO), $7 d$ after treatment (T1) and $14 d$ after treatment (T2). There were no significant differences in levels of inflammatory cytokines IL-6, IL-1 $\beta$, IL- 8 of patients in the two groups (P>0.05). After $7 \mathrm{~d}$ of treatment, levels of IL-6, IL-1 $\beta$, IL- 8 in the study group were all lower than the control group $(\mathrm{P}<0.05)$. After $14 \mathrm{~d}$ of treatment, levels of IL- 6 , IL- $1 \beta$ and IL- 8 were all lower than these of $7 d$ after treatment, and levels of IL-6, IL-1 $\beta$ and IL-8 were lower in the study group than the control group after $14 \mathrm{~d}$ of treatment $(\mathrm{P}<0.05)$ (Fig. 1).

Comparison of NIHSS scores of the two groups before and after treatment. NIHSS score was used to score neurological 
A

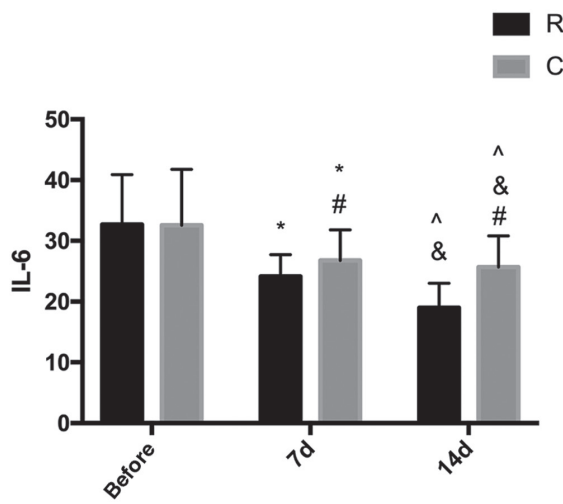

B

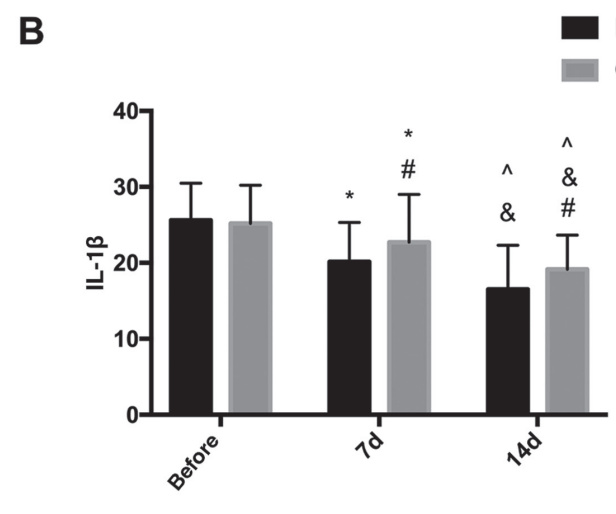

Research group Control group

C

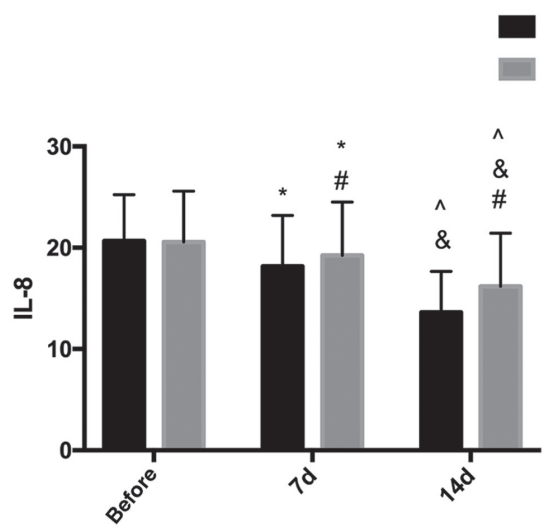

Research group

Control group

Figure 1. (A) Changes of inflammatory cytokine IL-6 level before treatment (T0), $7 \mathrm{~d}$ after treatment (T1) and $14 \mathrm{~d}$ after treatment of patients in the two groups. (B) Changes of inflammatory cytokine IL-1 $\beta$ level before treatment (T0), 7d after treatment (T1) and 14d after treatment of patients in the two groups. (C) Changes of inflammatory cytokine IL-8 level before treatment (T0), $7 \mathrm{~d}$ after treatment (T1) and $14 \mathrm{~d}$ after treatment of patients in the two groups. " $\mathrm{P}<0.05$ compared with before treatment; ${ }^{*} \mathrm{P}<0.05$ compared with study group; ${ }^{\wedge} \mathrm{P}<0.05,14 \mathrm{~d}$ after treatment compared with $7 \mathrm{~d}$ after treatment; ${ }^{\&} \mathrm{P}<0.0514 \mathrm{~d}$ after treatment compared with before treatment.

defects before and after surgery. The results showed that there was no significant difference in NIHSS score between the two groups before treatment $(\mathrm{P}>0.05)$. After treatment, NIHSS scores of the two groups at the four time periods ( $2 \mathrm{~h}, 1 \mathrm{~d}, 7 \mathrm{~d}$ and $14 \mathrm{~d}$ after treatment) were significantly lower than those before treatment, with statistically significant differences $(\mathrm{P}<0.05)$. The improvement of NIHSS score in the study group was significantly better than that in the control group, and the difference was statistically significant $(\mathrm{P}<0.05)$ (Fig. 2).
Research group

Control group

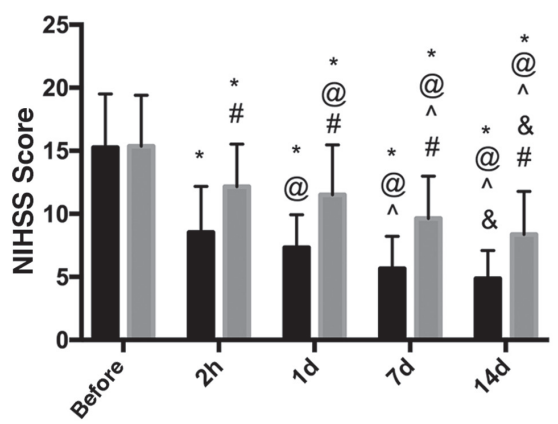

Figure 2. The NIHSS scores before treatment in four time periods $(2 \mathrm{~h}, 1 \mathrm{~d}, 7 \mathrm{~d}$ and $14 \mathrm{~d}$ ) after treatment in the two groups were compared. " $\mathrm{P}<0.05$ compared with before treatment; ${ }^{\circledR} \mathrm{P}<0.05$ compared with $2 \mathrm{~h}$ after treatment; ${ }^{\wedge} \mathrm{P}<0.05$ compared with $1 \mathrm{~d}$ after treatment; ${ }^{\circledR} \mathrm{P}<0.05$ compared with $7 \mathrm{~d}$ after treatment; " $\mathrm{P}<0.05$ compared with the study group of the same time.

Research group
Control group

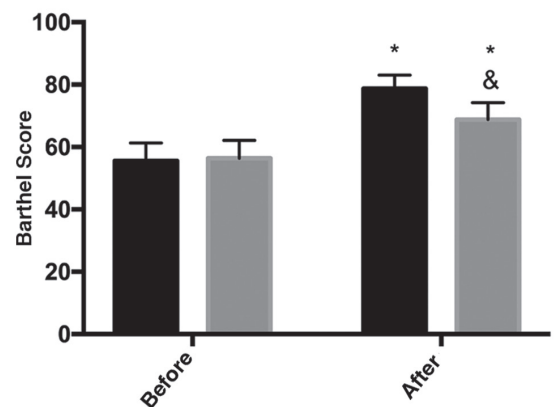

Figure 3. Comparison of Barthel index score between the two groups. ${ }^{*} \mathrm{P}<0.05$ compared with before treatment; ${ }^{\circledR} \mathrm{P}<0.05$ compared with study group.

Clinical efficacy of two groups of patients. The clinical efficacy of the two groups of patients was compared, and the results showed that the marked efficiency rate of the study group was significantly higher than that of the control group on $14 \mathrm{~d}$ after treatment $(\mathrm{P}<0.05)$, and the difference in the total efficiency of the two groups was not statistically significant $(\mathrm{P}>0.05)$ (Table II).

Comparison of Barthel index score between the two groups. There was no significant difference in Barthel index score between the two groups before treatment $(\mathrm{P}>0.05)$, while Barthel index score after treatment was higher than that of the control group $(\mathrm{P}<0.05)$ (Fig. 3).

Disease recurrence rate within 1 year in the two groups. Patients in the two groups were followed up for one year, and 120 patients were successfully followed up, with a follow-up success rate of $93.02 \%$. Among them, there were 3 patients lost to follow-up in the study group and 6 patients in the control group. The one-year disease recurrence rate of the two groups was compared, and it was lower in the study group than the control group $(\mathrm{P}<0.05)$ (Table III). 
Table I. Comparison of general data of patients in the two groups (\%).

\begin{tabular}{|c|c|c|c|c|}
\hline & Research group $(\mathrm{n}=69)$ & Control group $(\mathrm{n}=60)$ & $\operatorname{tor} \chi^{2}$ & P-value \\
\hline Age (years) & $61.6 \pm 4.5$ & $62.2 \pm 4.8$ & 0.732 & 0.465 \\
\hline \multicolumn{3}{|l|}{ Sex } & 0.305 & 0.581 \\
\hline Male & $48(69.57)$ & $39(65.00)$ & & \\
\hline Female & $21(30.43)$ & $21(35.00)$ & & \\
\hline BMI $\left(\mathrm{kg} / \mathrm{cm}^{2}\right)$ & $31.52 \pm 5.05$ & $30.86 \pm 4.72$ & 0.763 & 0.447 \\
\hline \multirow[t]{2}{*}{ Nitric oxide synthase (NOS) U/ml } & 0.821 & 0.413 & & \\
\hline & $24.16 \pm 3.58$ & $23.62 \pm 3.89$ & & \\
\hline \multirow[t]{2}{*}{ Endothelin-1 (ET-1) pg/ml } & 0.751 & 0.454 & & \\
\hline & $92.04 \pm 3.54$ & $91.57 \pm 3.55$ & & \\
\hline \multirow[t]{2}{*}{ Vascular endothelial growth factor (VEGF) $\mathrm{pg} / \mathrm{ml}$} & 0.572 & 0.569 & & \\
\hline & $306.65 \pm 32.87$ & $309.87 \pm 30.75$ & & \\
\hline \multirow{2}{*}{$50 \mathrm{sec}$ hemodynamics (SRV) $\mathrm{mPa} / \mathrm{sec}$} & 0.919 & 0.360 & & \\
\hline & $6.23 \pm 0.32$ & $6.17 \pm 0.42$ & & \\
\hline \multicolumn{2}{|l|}{ Living environment } & & 0.688 & 0.407 \\
\hline City & $59(85.51)$ & $48(80.00)$ & & \\
\hline Countryside & $10(14.49)$ & $12(20.00)$ & & \\
\hline \multicolumn{2}{|l|}{ Smoking history } & & 0.011 & 0.915 \\
\hline With & $34(49.28)$ & $29(48.33)$ & & \\
\hline Without & $35(50.72)$ & $31(51.67)$ & & \\
\hline \multicolumn{2}{|l|}{ Drinking history } & & 1.805 & 0.179 \\
\hline With & $38(55.07)$ & $40(66.67)$ & & \\
\hline Without & $31(44.93)$ & $20(33.33)$ & & \\
\hline \multicolumn{2}{|l|}{ Family medical history } & & 0.736 & 0.391 \\
\hline With & $9(13.04)$ & $5(8.33)$ & & \\
\hline Without & $60(86.96)$ & 55 (91.67) & & \\
\hline \multicolumn{2}{|l|}{ Ethnicity } & & 2.956 & 0.086 \\
\hline Han & $59(85.51)$ & $66(94.29)$ & & \\
\hline Minority & $10(14.49)$ & $4(5.71)$ & & \\
\hline
\end{tabular}

\section{Discussion}

Cerebrovascular occlusion is the main cause of cerebral infarction (18), its disability rate and death rate remain high (19). At present, the main clinical treatment for elder cerebral vascular occlusion is neurointerventional thrombolysis. Although neurointerventional thrombolysis has a significant therapeutic effect on vascular diseases (20), deficiencies of this method are also very significant. For example, if the puncture point and the puncture path are artificially selected, there may be deviations which have certain risks for the complicated tissue of the blood vessel. It not only causes secondary injury to the patient, but also causes more serious vascular occlusion (21). Therefore, how to accurately implement neurointerventional thrombolysis is the key and difficult point of treatment of cerebrovascular diseases. As shown in this study, treatment with 3D-DSA assisted neurointerventional thrombolysis is of great significance for future clinical practice.

The results showed that the levels of inflammatory cytokines IL-6, IL-1 $\beta$ and IL- 8 in the patients after 3D-DSA combined with neurointerventional thrombolysis were significantly lower than those in the patients treated with neurointerventional thrombolysis alone. This suggests that 3D-DSA combined with neurointerventional thrombolysis can reduce the inflammation caused by vascular occlusion in patients more effectively. Suzuki et al (22) aiming at the application of reconstruction techniques in the diagnosis and treatment of cerebral aneurysms have shown that 3D-DSA images are excellent for cerebral aneurysms, and the levels of inflammatory cytokines are well controlled after treatment. Therefore, it is speculated that 3D-DSA can make the puncture more accurate and the intervention channel better when performing cerebral vascular occlusion surgery, bringing less stress injury and, thus to reduce inflammatory cytokines. The NIHSS scores of the two groups of patients before and after treatment were compared, and the results showed that the improvement of NIHSS scores of the patients treated with 3D-DSA combined with neurointerventional thrombolysis was significantly better than that of the patients treated with neurointerventional thrombolysis alone. This suggests that the use of 3D-DSA assisted neurointerventional thrombolysis is more beneficial to the protection of neurological function and improvement of 
Table II. Comparison of clinical efficacy between the two groups.

\begin{tabular}{lccccccc}
\hline Groups & $\begin{array}{c}\text { No. of } \\
\text { cases }\end{array}$ & Cure & $\begin{array}{c}\text { Significant } \\
\text { effect }\end{array}$ & Effective & Invalid & Deterioration & $\begin{array}{c}\text { Marked } \\
\text { efficiency rate }\end{array}$ \\
\hline effective rate
\end{tabular}

Table III. Comparison of disease recurrence rate within 1 year in two groups.

\begin{tabular}{lcccc}
\hline & $\begin{array}{c}\text { Research } \\
\text { group } \\
(\mathrm{n}=66)\end{array}$ & $\begin{array}{c}\text { Control } \\
\text { group } \\
(\mathrm{n}=54)\end{array}$ & $\chi^{2}$ & P-value \\
\hline $\begin{array}{l}\text { Recurrence } \\
\text { Without }\end{array}$ & $\begin{array}{r}7(10.61) \\
\text { recurrence }\end{array}$ & $14(25.93)$ & 4.828 & 0.028 \\
\hline
\end{tabular}

prognosis of patients. Then the therapeutic effect of the two groups of patients was observed, and the marked effective rate of using 3D-DSA as an adjuvant therapy was significantly higher than that of the patients treated with neurointerventional thrombolysis alone, indicating that the use of 3D-DSA as an adjuvant therapy has good clinical value and is worthy of popularization. In assessing the accuracy and practicability of 3D-DSA and 3D-CT angiography for cerebral aneurysms Ishida et al (23) revealed that 3D-DSA has a higher diagnostic accuracy, which was conducive to carry out more effective follow-up treatment for the disease, with better therapeutic effect. This supports our experimental results. We speculate that in the treatment of senile cerebrovascular occlusion, it is precisely because 3D-DSA is detected at any angle in the brain, which greatly improves the detection efficiency, so that the neurointerventional thrombolysis can be performed more effectively and accurately, and the therapeutic effect can be improved. The study of Muruet et al (24) investigating neurointerventional thrombolytic therapy for ischemic stroke shows that thrombolytic therapy can improve long-term survival rate and functional status after ischemic stroke, its Barthel index is also consistent with this study. Whereas, in the present study, the curative effect of 3D-DSA- assisted neurointerventional thrombolysis is better than that of neurointerventional thrombolysis, so the Barthel index of patients under 3D-DSA-assisted therapy is also higher. The patients were followed up for one year, and the one-year disease recurrence rate of patients after 3D-DSA was significantly lower than that of patients treated with neurointerventional thrombolysis. This suggests that 3D-DSA adjuvant therapy has a good prognosis for senile cerebrovascular occlusion. In future clinical practice, patients with senile cerebrovascular occlusion can be treated by 3D-DSA combined with neurointerventional thrombolysis.

However, we did not make a comparative study on 3D-DSA combined with other therapeutic methods, and it is still unclear whether 3D-DSA combined with other therapeutic methods have the same efficacy in treating senile cerebrovascular occlusion. As the number of subjects in this study is small and the research period is short further study is required.

In conclusion, 3D-DSA combined with neurointerventional thrombolysis in patients with cerebrovascular occlusion can significantly reduce the expression of inflammatory cytokines, improve quality of life, and has a high clinical value.

\section{Acknowledgements}

Not applicable.

\section{Funding}

No funding was received.

\section{Availability of data and materials}

The datasets used and/or analyzed during the present study are available from the corresponding author on reasonable request.

\section{Authors' contributions}

SJ observed and compared the levels of inflammatory cytokines and wrote the manuscript. LG and $\mathrm{ZW}$ conceived and designed the study. $\mathrm{LZ}$ and $\mathrm{JH}$ were responsible for the collection and analysis of the experimental data. BT and SY interpreted the data and drafted the manuscript. SJ and ZW revised the manuscript critically for important intellectual content. All the authors read and approved the final manuscript.

\section{Ethics approval and consent to participate}

The study was approved by the Ethics Committee of Affiliated Hospital of Zunyi Medical University (Zunyi, China). Patients who participated in this research had complete clinical data. Signed informed consents were obtained from the patients or the guardians.

\section{Patient consent for publication}

Not applicable.

\section{Competing interests}

The authors declare that they have no competing interests. 


\section{References}

1. Horie N, Tateishi Y, Morikawa M, Morofuji Y, Hayashi K, Izumo T, Tsujino A, Nagata I and Matsuo T: Acute stroke with major intracranial vessel occlusion: characteristics of cardioembolism and atherosclerosis-related in situ stenosis/occlusion. J Clin Neurosci 32: 24-29, 2016.

2. Park MG, Yoon CH, Baik SK and Park KP: Susceptibility vessel sign for intra-arterial thrombus in acute posterior cerebral artery infarction. J Stroke Cerebrovasc Dis 24: 1229-1234, 2015.

3. Jayaraman MV,Hussain MS, Abruzzo T, Albani B, Albuquerque FC, Alexander MJ, Ansari SA, Arthur AS, Baxter B, Bulsara KR, et al: Embolectomy for stroke with emergent large vessel occlusion (ELVO): Report of the Standards and Guidelines Committee of the Society of NeuroInterventional Surgery. J Neurointerv Surg 7: 316-321, 2015.

4. Ferrer I and Vidal N: Neuropathology of cerebrovascular diseases. Handb Clin Neurol 145: 79-114, 2018.

5. Vedantham S, Piazza G, Sista AK and Goldenberg NA: Guidance for the use of thrombolytic therapy for the treatment of venous thromboembolism. J Thromb Thrombolysis 41: 68-80, 2016.

6. Lindekleiv H, Berge E, Bruins Slot KM and Wardlaw JM: Percutaneous vascular interventions versus intravenous thrombolytic treatment for acute ischaemic stroke. Cochrane Database Syst Rev: Oct 26,2018 (Epub ahead of print). doi: 10.1002/14651858. CD009292.pub2.

7. Tian Q, Zhu G and Dong S: Clinical efficacy of neurointerventional catheter thrombolysis for cerebral infarction. J Clin Nurs Res: Nov 30, 2019 (Epub ahead of print). doi: 10.26689/jenr. v3i6.867.

8. Lei X, Zheng H, Wang Y and Liu C: Observation of the clinical effect of interventional therapy of acute cerebral infarction with digital subtraction angiography. Chin J Primary Med Pharm 24: 3152-3155, 2017 (In Chinese).

9. Rao ACA, Shah SA, Sim BW, Yun ST, Jain NS, Kalani Y and Francis IC: Neuroradiological endovascular intervention for diplopia in a case of aneurysmal aberrant regeneration of the third nerve. Cureus 9: e1340, 2017.

10. Xianxian Z, Chengsong Y, Qiang M, Fei W, Lin S, Huiyan D and Zili G: The efficiency analysis of thrombolytic rt-PA combined with intravascular interventional therapy in patients with acute basilar artery occlusion. Int J Biol Sci 13: 57-64, 2017.

11. Taslakian B, Sebaaly MG and Al-Kutoubi A: Patient evaluation and preparation in vascular and interventional radiology: What every interventional radiologist should know (part 2: patient preparation and medications). Cardiovasc Intervent Radiol 39: 489-499, 2016.

12. Mine B, Tancredi I, Aljishi A, Alghamdi F, Beltran M, Herchuelz $M$ and Lubicz B: Follow-up of intracranial aneurysms treated by a WEB flow disrupter: A comparative study of DSA and contrast-enhanced MR angiography. J Neurointerv Surg 8: 615-620, 2016.

13. Papafaklis MI, Bourantas CV, Yonetsu T, Vergallo R, Kotsia A, Nakatani S, Lakkas LS, Athanasiou LS, Naka KK, Fotiadis DI, et al: Anatomically correct three-dimensional coronary artery reconstruction using frequency domain optical coherence tomographic and angiographic data: Head-to-head comparison with intravascular ultrasound for endothelial shear stress assessment in humans. EuroIntervention 11: 407-415, 2015.
14. Athanasiou L, Rigas G, Sakellarios AI, Exarchos TP, Siogkas PK, Bourantas CV, Garcia-Garcia HM, Lemos PA, Falcao BA, Michalis LK, et al: Three-dimensional reconstruction of coronary arteries and plaque morphology using CT angiography - comparison and registration with IVUS. BMC Med Imaging 16: 9, 2016.

15. Jeyaseelan RD, Vargo MM and Chae J: National Institutes of Health Stroke Scale (NIHSS) as an early predictor of poststroke dysphagia. PM R 7: 593-598, 2015.

16. Albers GW, Lansberg MG, Kemp S, Tsai JP, Lavori P, Christensen S, Mlynash M, Kim S, Hamilton S, Yeatts SD, et al: A multicenter randomized controlled trial of endovascular therapy following imaging evaluation for ischemic stroke (DEFUSE 3). Int J Stroke 12: 896-905, 2017.

17. Prodinger B, O'Connor RJ, Stucki G and Tennant A: Establishing score equivalence of the Functional Independence Measure motor scale and the Barthel Index, utilising the International Classification of Functioning, Disability and Health and Rasch measurement theory. J Rehabil Med 49: 416-422, 2017.

18. Buschmann EE, Hillmeister P, Bondke Persson A,Liebeskind DS Schlich L, Kamenzky R, Busjahn A, Buschmann IR, Bramlage P, Hetzel A, et al: Short-term external counterpulsation augments cerebral blood flow and tissue oxygenation in chronic cerebrovascular occlusive disease. Eur J Neurol 25: 1326-1332, 2018

19. Nerva JD and Levitt MR: Medical and surgical treatment of cerebrovascular occlusive disease. Principles Neurol Surg. Content Repository Only: 241-253. e3, 2018.

20. Pulicherla KK and Verma MK: Targeting therapeutics across the blood brain barrier (BBB), prerequisite towards thrombolytic therapy for cerebrovascular disorders - an overview and advancements. AAPS PharmSciTech 16: 223-233, 2015.

21. Tocci G and Presta V: Increased arterial stiffness and haemorrhagic transformation in ischaemic stroke after thrombolysis: A new marker of risk for cerebrovascular events and complications. Int J Cardiol 243: 471-472, 2017.

22. Suzuki K, Ikeda S, Ueda K, Nakamura T, Okabe M, Kadomura T, Baba R and Colbeth RE: Development of angiography system with cone-beam reconstruction using large-area flat-panel detector Proc SPIE 5368: 488-498, 2004.

23. Ishida F, Kawaguchi K, Mizuno M, Hoshino T, Murao K and Taki W: The accuracy and usefulness of 3D-DSA and 3D-CT angiography for cerebral aneurysms. Interv Neuroradiol 7 (Suppl 1): 181-186, 2001.

24. Muruet W, Rudd A, Wolfe CDA and Douiri A: Long-term survival after intravenous thrombolysis for ischemic stroke: A propensity score-matched cohort with up to 10 -year follow-up. Stroke 49: 607-613, 2018.

This work is licensed under a Creative Commons Attribution-NonCommercial-NoDerivatives 4.0 International (CC BY-NC-ND 4.0) License. 